\title{
A pilot study comparing ergonomics in laparoscopy and robotics: beyond anecdotes and subjective claims
}

\section{Li-Jen Kuo ( $\sim$ kuolijen@gmail.com )}

Division of Colorectal Surgery, Taipei Medical University Hospital, Taipei, Taiwan; Department of Surgery, School of Medicine, College of Medicine, Taipei Medical University, Taipei, Taiwan https://orcid.org/0000-0002-4865-325X

James Chi-Yong Ngu

Changi General Hospital

Yen-Kuang Lin

Taipei Medical University

Chia-Che Chen

Taipei Medical University

\section{Research article}

Keywords: Ergonomics, Fatigue, Minimally invasive surgery, Laparoscopy; Robot

Posted Date: September 26th, 2019

DOI: https://doi.org/10.21203/rs.2.15244/v1

License: (1) This work is licensed under a Creative Commons Attribution 4.0 International License. Read Full License

Version of Record: A version of this preprint was published at Journal of Surgical Case Reports on February 1st, 2020. See the published version at https://doi.org/10.1093/jscr/rjaa005. 


\section{Abstract}

Background: Minimally-invasive surgery has been shown to more demanding on the surgeon than open surgery. This can be manifested as physical fatigue and impaired dexterity, affecting the performance of the surgeon. In this study, we aimed to use hand dexterity and grip strength test as objective measures to compare the difference in surgeon fatigue associated with robotic and laparoscopic colorectal surgery.

Methods: A pilot study was conducted between February 2019 and May 2019, recruiting consecutive colorectal patients operated on by a single surgeon using laparoscopy or robotics. We used the Purdue Pegboard Test (PBT) to assess hand dexterity, and the Camry Electronic Handgrip Dynamometer to assess hand grip strength. Before each operative procedure, the surgeon was tasked to perform both tests in a standardized sequence. These tests were repeated two hours into surgery.

Results: Eighteen patients were operated on, including 10 robotic and 8 laparoscopic cases. Statistical analysis revealed no difference in dexterity or muscle fatigue after operating with the robot. In contrast, there was a significant difference in the hand grip strength of both hands after laparoscopic surgery $(\mathrm{P}=$ 0.04 and 0.02 , respectively).

Conclusions: Our results show that the resultant fatigue after laparoscopy affects both hands of the surgeon. In contrast, there was no difference in dexterity or muscle fatigue after operating with the robot. Given the demands of complex colorectal surgeries, robotics may be a means of optimizing surgeon performance by reducing fatigue.

\section{Background}

While minimally-invasive techniques have been introduced for various types of colorectal procedures, the widespread adoption of minimally-invasive surgery (MIS) has been hampered by the technical challenges associated with their implementation. Studies have shown that performing laparoscopic surgery imposes more physical stress on the surgeon than open surgery, $(1,2)$ and authors have also described how the resultant surgical fatigue can result in mental exhaustion, increased irritability, impaired surgical judgement, and reduced dexterity. $(3,4)$ Indeed, the benefits of laparoscopy to patients appear to come at the expense of their surgeons' well-being.

While numerous publications have failed to demonstrate an improvement in clinical outcomes after robotic surgery, one of the true advantages of robotics - ergonomics-remains unrefuted, but difficult to objectively quantify. Earlier studies evaluating surgical ergonomics have mainly utilized subjective assessments and self-reported measures like questionnaires. (5-7) In addition, there is also a sparsity of studies looking into the effect of fatigue affecting surgical performance. $(8,9)$ We aimed to use hand dexterity and grip strength test as objective measures to compare the difference in surgeon fatigue associate with robotic and laparoscopic colorectal surgery.

\section{Methods}


A pilot study was conducted between February 2019 and May 2019. Consecutive patients undergoing elective laparoscopic and robotics colorectal procedures by one of the authors (CCC) were included. The surgeon was board-certified and experienced in both laparoscopic and robotic surgery. Surgeries that were completed in less than two hours were excluded from the study. For the laparoscopic cases, the first surgeon would generally operate standing on the opposite side of the target anatomy. Instruments with pistol-grip handle configurations were used, including advanced energy devices. All the patients who were included in this study had written informed consent. The method of anonymized retrospective data retrieval fulfilled the institutional requirements for IRB exemption.

We used the Purdue Pegboard Test (PBT) to assess hand dexterity, and the Camry Electronic Handgrip Dynamometer to assess hand grip strength. Before each operative procedure, surgeon was tasked by a qualified occupational therapist (YHT) to perform the PBT in a standardized sequence. This was followed by the hand grip strength test for the right hand ( 5 times), and then left hand ( 5 times). Two hours into each surgery, the surgeon would take a technique break and repeat both tests in the same sequence. During the time of the test, another surgeon would continue the operation until the main surgeon completed the study protocol and returned to complete the surgery.

\section{Hand dexterity test}

The PBT includes four subtests involving the (i) dominant hand, (ii) non-dominant hand, (iii) both-hands, and (iv) an assembly subtest. In the first two subtests, the surgeon was given 30 seconds to pick up and insert pins into holes according to a fixed sequence. This task was completed using the dominant hand, followed by the non-dominant hand. In subtest (iii), the surgeon was given another 30 seconds to use both hands at the same time to repeat the earlier task. The number of pins in subtests (i) and (ii), and the number of pairs in subtest (iii) were recorded. Finally, in subtest (iv), the surgeon was allocated 60 seconds to use both hands alternatingly to assemble a pin, a washer, a collar, followed by another washer, in that specific sequence. The score of this subtest was determined by the number of pin-washercollar-washer correctly assembled.

\section{Hand grip strength test}

Grip strength was measured using the Camry Electronic Handgrip Dynamometer (Camry Scale). The test was performed with the surgeon gripping the dynamometer with the base rest on the first metacarpal, while the handle should rest on middle of the four fingers. The posture of the surgeon was standardizedseated with elbows by his side and flexed at 90 degrees, with his wrist in neutral position. Five measurements of grip strength were taken for each hand, and the mean value recorded.

\section{Statistical analysis}


SAS version 9 for Windows (SAS institute, Cary, NC) was used for data manipulation and assembly for analysis. The central tendency of fatigues measures were displayed using averages. The paired T-T test was used to investigate average differences before and after the operation while Mann-Whitney $U$ test was applied for variables that are not normally distributed. Corrections for multiple testing were also performed using Bonferroni post hoc multiple comparison tests. The differences were considered significant if the $P$ valve was smaller than 0.05 .

\section{Results}

Eighteen patients were operated on, including 10 robotics cases and 8 laparoscopic ones. Table 1 summarizes the patient demographic and operative details. All procedures were completed using the intended operative modality with no cases of conversion.

In the robotic group, the mean number of pegs placed in 30 seconds for the right hand prior to surgery was $13.5 ; 11.4$ for the left hand and 9.9 for both hands (Table 2). The mean number for assembling in 60 seconds was 7.8. The mean grip strength for the right and left hands were $36.9 \mathrm{~kg}$ and $31.1 \mathrm{~kg}$, respectively. The corresponding results after operative for 2 hours were 13.0, 10.9, 8.9, and 7.6. The mean grip strength for right hand was $36.1 \mathrm{~kg}$ and $30.6 \mathrm{~kg}$ for left hand. Statistical analysis revealed no difference in dexterity or muscle fatigue after operating with the robot. In the laparoscopic group, the mean number of pegs placed in 30 seconds for the right hand prior to surgery was $13.5 ; 11.5$ for the left hand and 9.6 for both hands. The mean number for assembling in 60 seconds was 8.2. The mean grip strength for the right and left hands were $37.7 \mathrm{~kg}$ and $32.3 \mathrm{~kg}$, respectively. The corresponding results after operating for 2 hours were 14.0,10.8,9.8, and 8.0. The mean grip strength for right hand was 35.0 $\mathrm{kg}$ and $29.0 \mathrm{~kg}$ for left hand. In contrast, there was a significant difference in the hand grip strength of right and left hand after laparoscopic surgery ( $P=0.04$ and 0.02 , respectively). Comparing individual test results between the robotic and laparoscopic groups showed no significant difference in dexterity and fatigue.

\section{Discussion}

The challenges of laparoscopy have been attributed to the short-comings in the design of conventional instruments, and the awkward posturing adopted by operators for protracted periods of surgery. Most laparoscopic instruments incorporate a ringed pistol-type handle that results in excessive flexion and deviation of the surgeon's wrist during tissue manipulation. The hand-to-tip force transmission is also less efficient compared to the instruments used in open surgery. $(10,11)$ The visual display of the operative field is also paramount to the conduct of laparoscopic surgery. While three-dimensional scope systems have been introduced in an attempt to address the lack of stereopsis, many of these still suffer from limitations, such as the loss of light intensity as a result of using polarized glasses. (12) Coupled with the need to constantly focus the image on a dynamically moving surgical instrument, a trained scope assistant is invaluable to the smooth conduct of complex laparoscopic surgeries. Robotic surgical systems have been designed to address these issues. Not only does the robotic system reduce the 
reliance on a trained assistant, it also provides greater surgeon comfort, autonomy and improved fluidity of surgical motion. (13-15) Newer generation scopes also provide superior image quality and depth perception.

Our results show that the amount of physical fatigue after laparoscopy is significantly more, affecting both hands of the surgeon. However, the dexterity and presumable overall performance of the surgeon was not impaired. This could be because the PBT did not require significant motor power to complete, and perhaps the hand-eye coordination of an experienced MIS surgeon was able to compensate for the loss of strength. Alternatively, the complexity of the cases performed laparoscopically might not have been enough to stress the surgeon, or this might not have been apparent after only two hours of operating, taking into account that the timing also included less demanding tasks like port placement. Conversely, it could be argued that the use of robotics mitigated the amount of fatigue experienced by the surgeon even in complex colorectal operations, given that the majority of the robotic cases were either low anterior resection or transanal procedures. Given the demands of complex colorectal surgeries and high-volume workload, robotics may be a means of optimizing surgeon performance by reducing fatigue. (16) This would expectedly help to reduce iatrogenic injuries and mistakes caused by human error, and possible justify the added expense of robotic technology.

As this was a pilot study evaluating the feasibility of such a method of assessment, out analysis inevitable suffers from the limitations of a small sample size. In addition, operator posture and arm positions during surgery were not recorded, although these were assumed to have been ergonomically ideal given the experience of the surgeon studied. While the study of a single surgeon's ergonomics allowed comparability across the two MIS platforms, it also limited the generalizability of our findings. The results may also differ from surgeons who utilize different laparoscopic and robotic equipment from our institution.

\section{Conclusions}

Our results show that the resultant fatigue after laparoscopy affects both hands of the surgeon. In contrast, there was no difference in dexterity or muscle fatigue after operating with the robot. Given the demands of complex colorectal surgeries, robotics may be a means of optimizing surgeon performance by reducing fatigue.

\section{Abbreviations}

\section{MIS = minimally invasive surgeryखPBT = Purdue Pegboard Test}

\section{Declarations}

- Ethics approval and consent to participate: All the patients who were included in this study had written informed consent. The method of anonymized retrospective data retrieval fulfilled the 
institutional requirements for IRB exemption.

- Consent for publication: Yes

- Availability of data and material: Not applicable

- Competing interests: The authors declare that they have no competing interests

- Funding: The authors declare that they have no funding for the research reported

- Authors' contributions:

\section{LJK: original idea creator and manuscript writing}

\section{JCN: data interpretation and participate manuscript writing}

\section{YKL: data interpretation and statistics analysis}

\section{CCC: participate in operative procedure}

- Acknowledgements: Not applicable

\section{References}

1. Berguer R, Smith WD, Chung YH. Performing laparoscopic surgery is significantly more stressful for the surgeon than open surgery. Surg Endosc 2001; 15:1204-7.

2. Berguer R, Rab GT, Abu-Ghaida H, Alarcon A, Chung J. A comparison of surgeons' posture during laparoscopic and open surgical procedures. Surg Endosc 1997; 11:139-42.

3. Cuschieri A. Whither minimal access surgery: tribulations and expectations. Am J Surg 1995; 169:919.

4. Grissinger M. An Exhausted Workforce Increases the Risk of Errors. PT 2009; 34:120-3.

5. Law KE, Lowndes BR, Kelley SR, Blocker RC, Larson DW, Hallbeck MS, Nelson H. NASA-Task Load Index Differentiates Surgical Approach: Opportunities for Improvement in Colon and Rectal Surgery. Ann Surg. 2018 Dec 29. doi: 10.1097/SLA.0000000000003173.

6. Moore LJ, Wilson MR, McGrath JS, Waine E, Masters RS, Vine SJ. Surgeons' display reduced mental effort and workload while performing robotically assisted surgical tasks, when compared to conventional laparoscopy. Surg Endosc 2015; 29:2553-60.

7. Dias RD, Ngo-Howard MC, Boskovski MT, Zenati MA, Yule SJ. Systematic review of measurement tools to assess surgeons' intraoperative cognitive workload. Br J Surg 2018; 105:491-501.

8. Sturm L, Dawson D, Vaughan R, Hewett P, Hill AG, Graham JC, Maddern GJ. Effects of fatigue on surgeon performance and surgical outcomes: a systematic review. ANZ J Surg 2011; 81:502-9. 
9. McCormick F, Kadzielski J, Landrigan CP, Evans B, Herndon JH, Rubash HE. Surgeon fatigue: a prospective analysis of the incidence, risk, and intervals of predicted fatigue-related impairment in residents. Arch Surg 2012; 147:430-5.

10. Berguer R. Surgical technology and the ergonomics of laparoscopic instruments. Surg Endosc 1998; 12:458-62.

11. Berguer R, Forkey DL, Smith WD. Ergonomic problems associated with laparoscopic surgery. Surg Endosc 1999; 13:466-8.

12. Schwab K, Smith R, Brown V, Whyte M, Jourdan. Evolution of stereoscopic imaging in surgery and recent advances. World J Gastrointest Endosc 2017; 9:368-77.

13. Leal Ghezzi T, Campos Corleta O. 30 Years of Robotic Surgery. World J Surg 2016; 40:2550-7.

14. Köckerling F. Robotic vs. Standard Laparoscopic Technique - What is Better? Front Surg 2014; 1:15.

15. Van Koughnett JA, Jayaraman S, Eagleson R, Quan D, van Wynsberghe A, Schlachta CM. Are there advantages to robotic-assisted surgery over laparoscopy from the surgeon's perspective? J Robot Surg 2009; 3:79-82.

16. Hurley AM, Kennedy PJ, O'Connor L, Dinan TG, Cryan JF, Boylan G, O'Reilly BA. SOS save our surgeons: Stress levels reduced by robotic surgery. Gynecol Surg 2015; 12:197-206.

\section{Tables}

Due to technical limitations, tables are only available as a download in the supplemental files section

\section{Supplementary Files}

This is a list of supplementary files associated with this preprint. Click to download.

- Table2.docx

- Table1.docx 